\title{
Pseudoband formation: Missed ATFL tears on MR imaging
}

\author{
Vajid Khan ${ }^{1}$, Jatin Kaicker ${ }^{1}$, James Namburi ${ }^{1}$, Pawel Stefanksi ${ }^{1}$, Bradley Petrisor ${ }^{2}$, Hema Choudur $^{{ }^{*}}$ \\ ${ }^{1}$ Department of Medical Imaging, McMaster University, Hamilton, Canada \\ ${ }^{2}$ Department of Orthopedic Surgery, McMaster University, Hamilton, Canada \\ Email: *choudur@hhsc.ca
}

Received 20 July 2013; revised 20 August 2013; accepted 28 August 2013

Copyright (C) 2013 Vajid Khan et al. This is an open access article distributed under the Creative Commons Attribution License, which permits unrestricted use, distribution, and reproduction in any medium, provided the original work is properly cited.

\begin{abstract}
Background: The anterior talofibular ligament (ATFL) is the ligament most commonly injured during ankle sprains. Objective: The purpose of this investigation was to assess the sensitivity of Magnetic Resonance (MR) imaging for ATFL with open surgery, Brostrom-Gould surgical interventional considered the gold standard. Methods: A retrospective chart review using Operating Room (OR) and MR reports from April 2008-November 2009 led to a review of 137 charts, with 15 cases meeting the inclusion criteria. Results: The sensitivity for ATFL tears irrespective of extent of the tear was 93\%. For full thickness ATFL tears, 10 were found to be positive on MR but 13 on OR with a sensitivity of $77 \%$. Of the three missed cases, 2 were described as sprain injuries and the other normal using MRI. Conclusion: The recommendations for MR assessment of ankle injury include use of dedicated sequences and understanding the "pseudoband" concept. The two full thickness and two partial thickness tears on OR described as sprain injuries on MRI had a band of inflammatory granulation tissue. This tissue accounted for the appearance of the thick continuous band at MRI, the pseudoband. This study reaffirms the usefulness of MR in determining ATFL tears, aiding surgical decision making.
\end{abstract}

Keywords: ATFL Tear; Brostrom-Gould Surgery; Pseudoband

\section{INTRODUCTION}

Ankle sprains are the most common injuries accounting for $20 \%$ of sport injuries in the United States [1]. The most common ligaments injured are the anterior talofibular ligament (ATFL) and calcaneofibular ligament

\footnotetext{
*Corresponding author.
}

(CFL). The ATFL is the most commonly torn as it is the weakest one [1]. The CFL is next in order of frequency and is almost always seen with an associated ATFL injury (Figures 1 and 2).

ATFL tears can be either full or partial thickness tears. Full tears that are acute have absent ligament or a complete cleavage of the ligament with pooling of fluid in the region (Figure 3). As the tear progresses with time, inflammatory granulation tissue, scar fill in and around the torn portion, bridging the tear. This mimics an intact ligament on MR. We have termed this entity as a "Pseudoband” (Figure 4). The pseudoband has margins that are irregular or blurred. The band is inhomogenous, attenuated and lax. It may be thick or thin as compared to the normal ligament depending on the duration of the tear. Furthermore, chronic full thickness tears can present an absent ligament or hypoplastic ligament remnant with attenuated MR T1/T2 signal and laxity. A partial thickness tear can be a partial discontinuity of fibers or focal thinning in any portion of the ligament with less fluid seen around the tear as opposed to a full thickness tear. On MR imaging, a sprain injury can appear as a normal ligament with surrounding inflammatory high signal or can appear as a thickened smoothly marginated low T1/T2

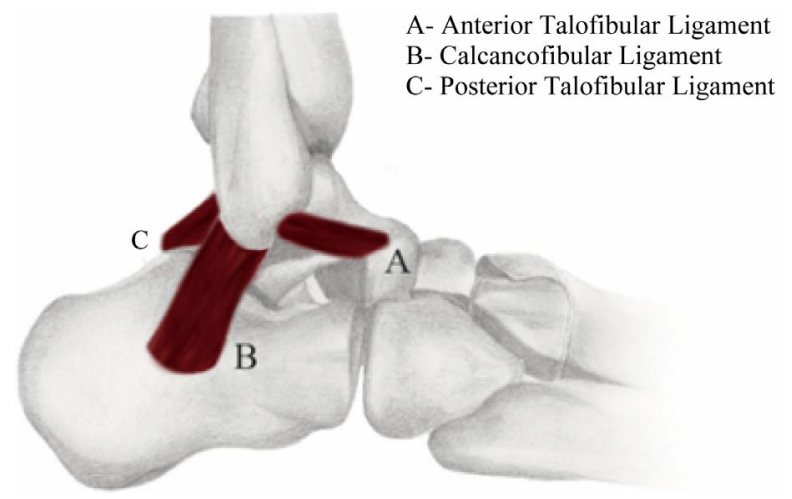

Figure 1. Ligaments in the foot. 


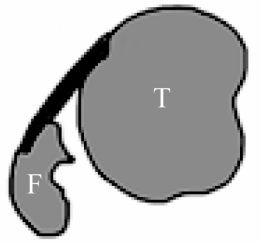

A

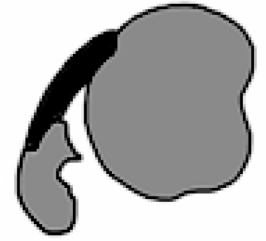

B

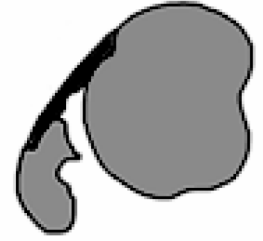

C

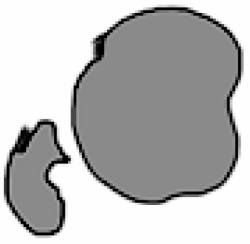

F

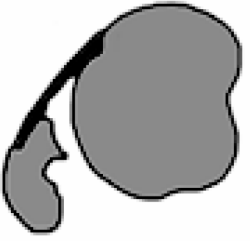

$\mathrm{D}$

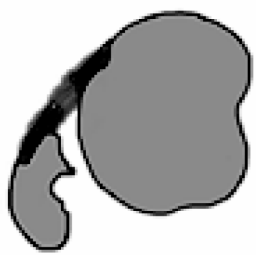

G
F: Absent Ligament (Rupture)

G: Pseudo Band Formation

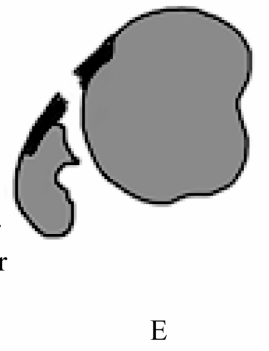

Figure 2. Injuries to the ATFL.

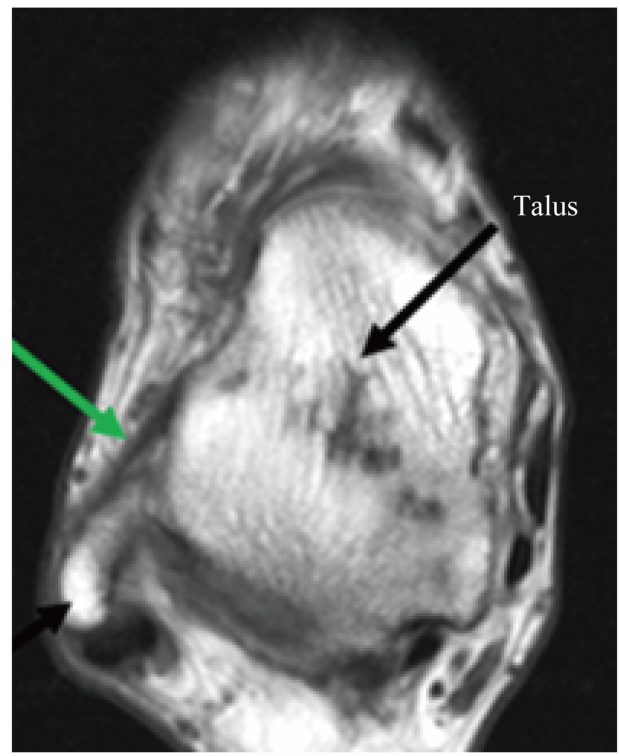

Figure 3. Normal ATFL (green) on an axial T1 weighted image. Fibula (black arrow).

structure with or without surrounding inflammatory signal, depending on the duration of the injury.

With MR imaging being a key modality in the diagnosis of ligament tears and sprains, it is important to understand the ability of MR imaging to detect ATFL injury and direct surgical management. The purpose of this investigation was to assess the sensitivity of MR imaging for ATFL at two hospitals in Hamilton, Ontario, Canada. In addition, the purpose is to discuss to "pseudoband" concept and recommend the utility of specific imaging sequences for more sensitive detection of ATFL tear.

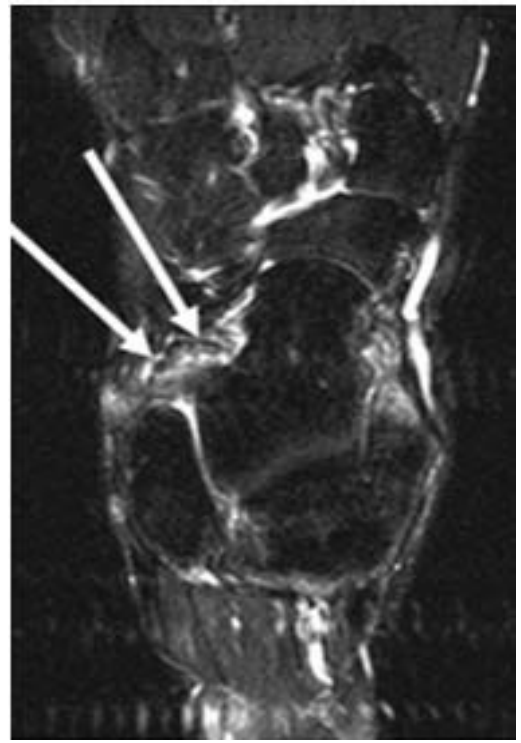

Figure 4. Axial T2 Fat suppressed sequence demonstrating pseudoband formation. The pseudoband is lax, irregular, inhomogenous and attenuated in signal intensity. This patient had a full thickness ATFL tear at surgery.

\section{MATERIALS AND METHODS}

\subsection{Data Abstraction}

This investigation was conducted as a retrospective review of reports of MR ankle studies from April 2008November 2009 referred to our department for ankle instability with or without pain. The MR reports were obtained from the Radiology Picture Archiving and Com- 
munication System (PACS), common to the two hospitals of the Hamilton Health Sciences. The MRs used at both sites were 1.5 Tesla Siemens Symphony Avanto MRI Scanners. All positive ankle injury cases are referred to an ankle orthopedic surgeon. Those MR reports that were positive or negative for ATFL were screened for subsequent surgery by the ankle orthopedic surgeon at our hospitals. Only those MR cases that had undergone open surgery for ankle instability between 6 months to one year of injury were included in our study. The medical charts of the selected patients were reviewed to assess for clinical symptoms and signs of instability prior to MRI and to the surgery. The MR ankle protocol for ankles is site specific. A total of 137 MRI cases were reviewed, with 15 cases meeting the inclusion criteria established for the investigation. Three musculoskeletal radiologists involved in reporting these MR cases had more than 8 years of experience at the time of reporting. There was no consensus reading. The ankle orthopedic surgeon who performed all the surgeries had 10 years of experience at the time of the surgery. The study was approved by the Ethics Review Board of Hamilton Health Sciences. For surgical intervention to be considered, clinical assessment of the ankle instability was the chief deciding factor and this decision was reinforced by the MR report of a tear and its extent. The surgical criteria used for operating was: ankle instability and full thickness tear on MR or ankle instability and high grade partial thickness tear on MR. The main determining factor for surgical intervention in cases where MRI did not concur with the clinical examination was clinical instability.

\subsection{Inclusion Criteria}

Patients were included in this study if they had presented to the ER with an ankle injury, regardless of age (except pediatric patients) and gender. The next criteria involved ensuring that the patients had the MRI by referral from the ankle orthopedic surgeon for clinical ankle instability, 3 - 6 months after injury. OR must have followed the MR with ATFL tears found during surgery. Results of OR and MR were compared. Sprains and tears of these ligaments were included in the study. The tears were subdivided as full thickness and partial thickness tears.

\subsection{Exclusion Criteria}

Patients were excluded from the investigation if an MR was performed with no subsequent surgery or if there was initial surgical intervention before the MR. Patients with significant ankle reconstruction or other potential confounders for viewing the ankle MR were also excluded.

\section{RESULTS}

A total of 15 patients were included in this investigation. The total number of ATFL injuries irrespective of extent of injury found 14 cases positive at MR but 15 at OR accounting for a sensitivity of $93 \%$. Tears were then assessed for being either full or partial thickness ATFL tears. For full thickness ATFL tears, 10 were found to be positive on MR but 13 on OR presentation, accounting for a sensitivity of $77 \%$. Of the three cases that were false negative at MR, 2 were described a sprain and in third case, the ligament was described as normal at MRI. The two high grade partial thicknesses ATFL tears at OR were described as sprain injuries on MR. The cases that were described as sprain injuries on MR but were identified as full thickness or high grade partial thickness tears at OR had one common feature. This was the presence of granulation tissue and debris or scar with debris closely surrounding and bridging the gap created by the tear, giving the appearance of a continuous band but being lax on application of pressure (Figure 5).

\section{DISCUSSION}

MRI is a standard imaging modality for the diagnosis of ATFL tears. It aids the orthopedic surgeon in treatment planning. Full thickness tears of the ATFL are associated with clinical instability. When attempting to examine the ATFL, axial imaging is the most useful. In this investigation, in most cases, axial oblique sequences were used in the plane of the ATFL decreasing the slice thickness to 3 $\mathrm{mm}$ with zero slice gap. As the images are acquired in the plane of the ligament with thinner slice thickness and zero slice gap, the tears are better delineated on these sequences. This ligament is recognized as a thin, uniformly low T1 and T2 signal band that runs from the anterior distal fibular tip to the talus [2]. On the same axial imaging plane, PTFL is seen as a relatively thicker uniformly low T1 and T2 band extending from inner distal fibular tip to the adjacent posterior talus [2]. It is seen deeper to the peroneal tendons, skirting the lateral calcaneus. It is better delineated in the coronal plane. The PTFL is well delineated on the coronal and axial planes.

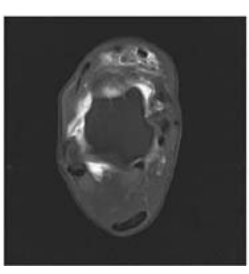

(a)

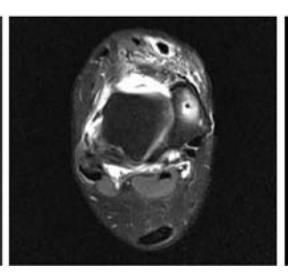

(b)

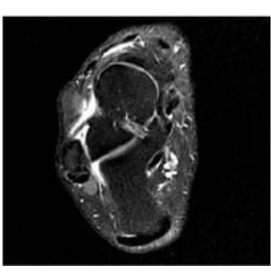

(c)
Figure 5. Example of axial PD fat suppressed (a) and dedicated axial oblique sequences (b) \& (c) in the plane of the ATFL sequences. The ATFL is clearly depicted on these sequences. Note the full thickness tear in image (b). 
In this study, a discrepancy was noted for 5 of the ATFL injuries seen at surgery. Two full thickness tears and two high grade partial thickness tears were not found on MR but discovered in the OR. Additionally, one full thickness tear at OR was found to be completely normal onMR. The two cases of the full thickness tears and the two cases of high grade partial thickness tears at OR were described as sprain injuries on MR Imaging. These cases may have been visualized as a ligament sprain due to the formation of a pseudoband. A pseudoband appears on MR as a thickened ligament with irregular margins and inhomogenous in signal intensity. This is in contrast to sprain injuries that have a smooth surface and are of low, homogenous signal.

One case of a full thickness tear at OR was described as a normal ATFL at MR and is a true miss. In this case, it was discovered that only an axial PD fat sequence and not an axial oblique PD fat sequence in the plane of the ATFL was acquired for imaging this ligament. Presumably, with these dedicated sequences, the radiologist would have had better visualization of the small full thickness ATFL tear given the thinner slices in the plane of the ligament with no slice gap. Furthermore, there was a large time interval (183 days) between MR examination and the surgery (Figure 6). During this period, interval re-injury may have occurred with the tear revealed in the OR.

Previous studies have described ligament injury. While ligament injury can heal as early as 7 days post injury, in some cases there can be delayed healing. If the sprain is close to the bone attachment, the probability of delayed healing is greater [3]. In some cases, irregular ligament margins can be seen up to 8 weeks post injury [3]. The improvement that occurs over time is due to remodeling of the fibrous scar as the resolving hematoma and collagen become more organized [3]. Such remodeling changes have been found to occur as much as 40 weeks

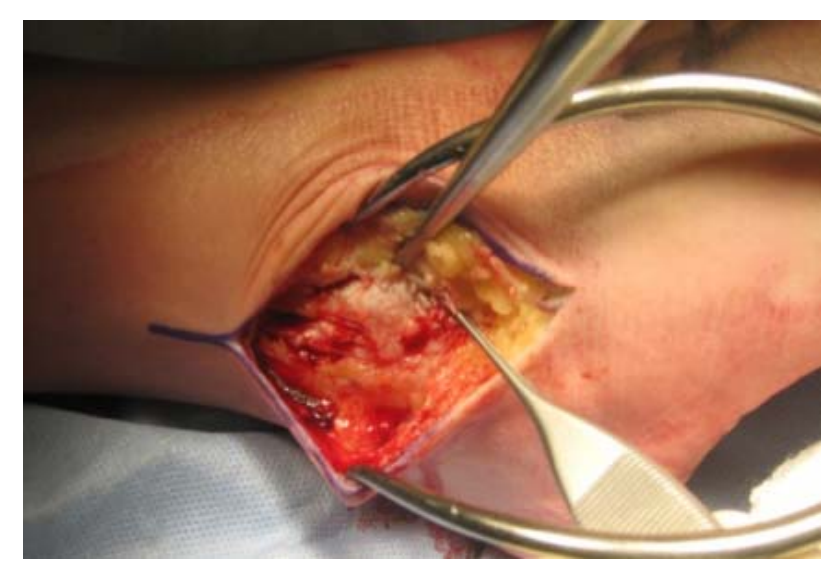

Figure 6. Open surgical intervention for torn ATFL. Intra-operative procedure demonstrates the torn ATFL between the forceps. post injury and seen on MRI scans. This investigation further demonstrates this concept, describing the "pseudoband" formation.

From this study, we make some recommendations for improving the MR assessment of ankle injury for clinically suspected ATFL tears. The first is to ensure an adequate clinical history and focused clinical patient assessment for detection of ankle instability which helps to tailor the MR protocol [4]. The second is to optimize the MR protocol to include dedicated sequences routinely in cases of ankle instability. Axial oblique PD fat sat in the plane of the ATFL can help improve visualization of this ligament and ability to detect and assess tears. It would be beneficial to study the cost effectiveness of dedicated sequences to determine if they affect outcomes as this was not addressed in our study. This is particularly important where clinical judgment favors a tear and routine MR protocol fails to confirm the tear as in one case in our study. The authors hope that this pilot study can be the initial stimulus in improving patient outcomes in ATFL tears by appropriate use of the aforementioned dedicated sequences (Table 1).

Prior studies described in literature have examined ATFL using radiography, MRI and ultrasonography $[5,6]$. Arthroscopy was used as the gold standard in these studies as compared to the open Brostrom-Gould type procedure in our study. While our study provided unique insight into the sensitivity of MR at detecting AFTL (both full and partial thickness) tears in comparison to surgery, there are some limitations. The sample size was small. The study was retrospective, and therefore, the musculoskeletal radiologists were not blinded to clinical information regarding instability. The history was not reviewed to ensure no injury in the interim between the MRI scan and surgery. This study involved two different hospitals, with differing MR protocols. Varying terminology was used by the radiologists and the orthopedic surgeon to describe the tear on the MRI and OR reports. It is recommended that a future study be accomplished in a prospective manner, with one, standardized protocol for MR imaging, preferably using the dedicated ATFL sequences, a larger sample size and standard nomenclature for describing ligament injury by the radiologists and orthopedic surgeons. This investigation has reaffirmed the usefulness of MR in determining ATFL tears. A combination of both tears lead to ankle instability for patients (Table 2).

\section{CONCLUSION}

In summary, accurate detection of the tear and definition of its extent on MR is of value to the orthopedic surgeon to decide whether or not to resort to surgical intervention. In cases of MR and OR mismatch, clinical instability is the key deciding factor. This study leads to the description 
Table 1. Ankle MR protocol at Hamilton General Hospital and Juravinksi Hospital.

\begin{tabular}{ll}
\hline Ankle protocol at HGH & Ankle Protocol at Juravinksi \\
\hline Ax T1 & Axial TSE PD Fat Sat \\
Ax TSE T2/PD with FS & - \\
Sag T1 & Sag TSE T1 \\
Sag STIR & Sag STIR \\
Sag TSE T2 & \\
Cor T1 & Cor TSE PD FS (small FOV) \\
Cor T2 FS & \\
Axial Oblique TSE PD FS & Axial Oblique TSE PD FS \\
\hline
\end{tabular}

Table 2. Sensitivity values of ATFL using MRI.

\begin{tabular}{lccc}
\hline Nature of Ankle Injury & $\begin{array}{c}\text { MRI } \\
\text { Positive }\end{array}$ & $\begin{array}{c}\text { MRI } \\
\text { Negative }\end{array}$ & $\begin{array}{c}\text { Sensitivity } \\
\text { Found }\end{array}$ \\
\hline $\begin{array}{l}\text { Full thickness ATFL tears } \\
\text { High grade partial thickness }\end{array}$ & $10 / 13$ & $3 / 13$ & $77 \%$ \\
$\begin{array}{l}\text { ATFL tears } \\
\begin{array}{l}\text { ATFL injury irrespective of } \\
\text { extent (tear or strain) }\end{array}\end{array}$ & $0 / 2$ & $2 / 2$ & $0 \%$ \\
\hline
\end{tabular}

of what we have termed "pseudobands", a novel concept which helps to explain the cause of false negative MR. The authors hope that developing familiarity with the MR imaging of "pseudobands" will lead to greater accuracy in identifying ATFL full thickness and high grade partial thickness tears.

\section{ACKNOWLEDGEMENTS}

The authors would like to thank Akhila Rachakonda of Hillfield Strathallan College for the line diagrams. The authors would also like to thank Mary Lou Schmuck from McMaster University for her assistance with statistical analysis.

\section{REFERENCES}

[1] Baert A.L., Reiser, M.F., Hricak, H. and Knauth, M. (2011) Sports injuries in children and adolescents. Springer, London, 219-222.

[2] Linklater, J. (2004) Ligamentous, chondral, and osteochondral ankle injuries in athletes. Semin Musculoskelet Radiol, 8, 81-98. doi:10.1055/s-2004-823016

[3] Labovitz, J.M., Schweitzer, M.E., Larka, U.B. and Solomon, M.G. (1998) Magnetic resonance imaging of ankle ligament injuries correlated with time. JAMPA, 88, 388393.

[4] Bickley, L.S. (2009) Bates guide to physical examination. Lippincott, Philadelphia, 2009.

[5] Oae, K., Takao, M., Uchio, Y. and Ochi, M. (2010) Evaluation of anterior talofibular ligament injury with stress radiography, ultrasonography and MR imaging. Skeletal Radiology, 39, 41-47. doi:10.1007/s00256-009-0767-x

[6] Park, H.J., Ca, S.D., Kim, S.S., Rho, M.H., Kwag, H.J., Park, N.H. and Lee, S.Y. (2012) Accuracy of MRI findings in chronic lateral ankle ligament injury: Comparison with surgical findings. Clinical Radiology, 67, 313-318. doi:10.1016/j.crad.2011.08.025 